\title{
Image-guided navigation surgery for bilateral choanal atresia with a Tessier number 3 facial cleft in an adult
}

Ji Yoon Sung ${ }^{1}$,

Kyu-Sup $\mathrm{Cho}^{2}$,

Yong Chan $\mathrm{Bae}^{1,3}$, Seong Hwan Bae ${ }^{1}$

${ }^{1}$ Department of Plastic and Reconstructive Surgery, Pusan National University School of Medicine, Busan; ${ }^{2}$ Department of Otorhinolaryngology and Biomedical Research Institute, Pusan National University Hospital, Pusan National University School of Medicine, Busan; ${ }^{3}$ Biomedical Research Institute, Pusan National University Hospital, Busan, Korea
The coexistence of craniofacial cleft and bilateral choanal atresia has only been reported in three cases in the literature, and only one of those cases involved a Tessier number 3 facial cleft. It is also rare for bilateral choanal atresia to be found in adulthood, with 10 previous cases reported in the literature. This report presents the case of a 19-year-old woman with a Tessier number 3 facial cleft who was diagnosed with bilateral choanal atresia in adulthood. At first, the diagnosis of bilateral choanal atresia was missed and septoplasty was performed. After septoplasty, the patient's symptoms did not improve, and an endoscopic examination revealed previously unnoticed bilateral choanal atresia. Computed tomography showed left membranous atresia and right bony atresia. The patient underwent an operation for opening and widening of the left choana with an image-guided navigation system (IGNS), which enabled accurate localization of the lesion while ensuring patient safety. Postoperatively, the patient became able to engage in nasal breathing and reported that it was easier for her to breathe, and there were no signs of restenosis at a 26-month follow-up. The patient was successfully treated with an IGNS.

Keywords: Choanal atresia / Computer-assisted / Craniofacial abnormalities / Face / Imaging, three-dimensional / Nasal obstruction / Nose / Otolaryngology / Surgery, plastic

\section{INTRODUCTION}

In 1976, Tessier classified facial clefts from type 0 to 14 based on anatomical considerations [1]. Tessier number 3 clefts, which are among the most complex and severe congenital facial anomalies, extend from the philtrum of the lip to the medial canthus, with foreshortening of this distance. Although facial cleft may be accompanied by complex congenital anomalies involving the bone and soft tissue, the coexistence of craniofacial cleft and bilateral choanal atresia is rare, with only three cases

Correspondence: Seong Hwan Bae

Department of Plastic and Reconstructive Surgery, Pusan National University

School of Medicine, 179 Gudeok-ro, Seo-gu, Busan 49241, Korea

E-mail: denev96@gmail.com

Received November 7, 2019 / Revised December 19, 2019 / Accepted January 1, 2020 reported in the literature [2-4].

Choanal atresia was first described by Roederer in 1775 . Congenital choanal atresia describes a narrowing of the anterior or posterior nasal apertures, which may be membranous $(10 \%)$ or bony (90\%), and the ratio of unilateral or bilateral cases is 3:2 [5]. Bilateral choanal atresia often presents as a respiratory emergency in newborns; therefore, it is rarely found in adults, with only 10 such cases reported in the literature [6-9].

Image-guided navigation systems (IGNS) are increasingly acknowledged as a useful technology for surgery. These systems provide real-time surgical navigation using preoperative computed tomographic (CT) images. During surgery, when the surgeon selects a point from the patient's anatomy using the tip of a probe, the corresponding points on the CT images are displayed 
on the monitor. Thus, these systems enable surgeons to perform minimally invasive procedures and assist in the identification of anatomic landmarks in difficult cases, thereby improving patient safety [10]. IGNS are used in neurosurgery, orthopedic surgery, otolaryngology, and craniomaxillofacial surgery, especially for brain tumors, vascular abnormalities, complex skull base surgery, and endoscopic operations such as endoscopic sinus surgery.

In this case study, we present the first case of bilateral choanal atresia found in adulthood in a patient with a Tessier number 3 facial cleft. The patient was successfully treated with IGNS.

\section{CASE REPORT}

A female patient was diagnosed with a Tessier number 3 facial cleft at birth. She underwent several surgical procedures for cleft lip and palate, cleft lip nasal deformity, and left nasal alar deformity starting at the age of 1 month at other clinics. From the age of 6 years, she was followed at our outpatient clinic and underwent several corrective operations for cleft lip nasal deformity, left nasal alar deformity, right lower eyelid deformity, alveolar cleft, and right exophthalmos.

At the age of 19 years, the patient wanted a further evaluation for nasal obstruction and nasal discharge, and was therefore referred to the department of otolaryngology. At first, the diagnosis of bilateral choanal atresia was missed on the initial craniofacial CT and intranasal endoscopy, because it was masked by severe septal deviation (Fig. 1). A septoplasty was done, but the

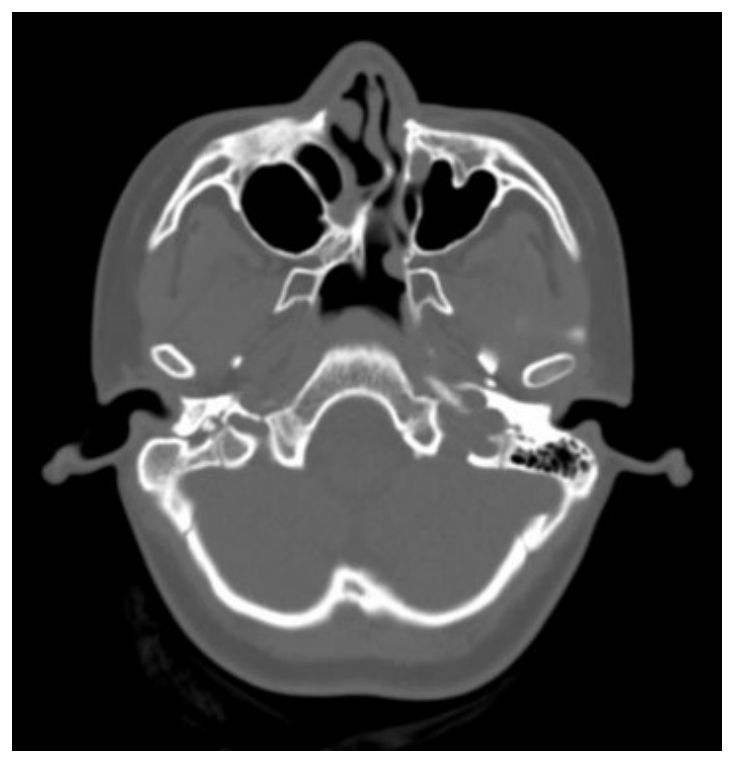

Fig. 1. Computed tomographic scan before septoplasty showing severe septal deviation. patient showed no signs of improvement.

After septoplasty, an endoscopic examination revealed previously unnoticed bilateral choanal atresia. Craniofacial CT revealed left membranous atresia and right bony atresia. On the right side, the bone was very thick, and it did not appear possible to open the right bony atresia. Therefore, endoscopic sinus surgery was planned to open and widen the left membranous choana using an IGNS (StealthStation ENT Navigation System; Medtronic, Minneapolis, MN, USA). One day before surgery, a 26-G lumbar puncture needle was used to make a puncture from the left intranasal side to the mouth under intranasal endoscopy to indicate where the left membranous choana should be (Fig. 2). After insertion of the lumbar puncture needle, transoral laryngoscopy was performed to find the lumbar puncture needle from the palatal side, making it possible to identify the exact site where the choana should be opened at the atresia plate.

Under general anesthesia, left choanal atresia was reconfirmed through intranasal endoscopy and the exact location was identified using the IGNS (Fig. 3). An injection of 1\% lidocaine with 1:100,000 epinephrine was made into the mucosa covering the posterior septum, atresia plate, and lateral nasal wall. After initial penetration of the atresia plate with a sickle knife, a microdebrider was used to remove the membranous mucosa over the left choana. To prevent restenosis, an 18-Fr Foley catheter was inserted through the left choana as a stent, and it was kept in place for 8 weeks.

The patient was discharged on postoperative day 2 and no ev-

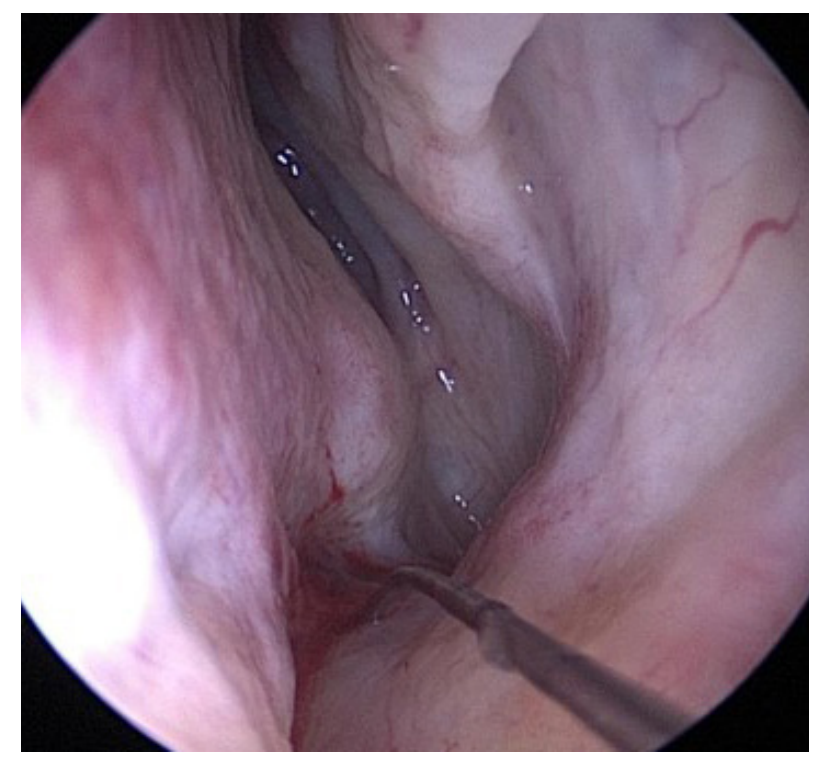

Fig. 2. Localization of the exact site for surgery by puncturing the site with a spinal puncture needle under intranasal endoscopy before surgery. 

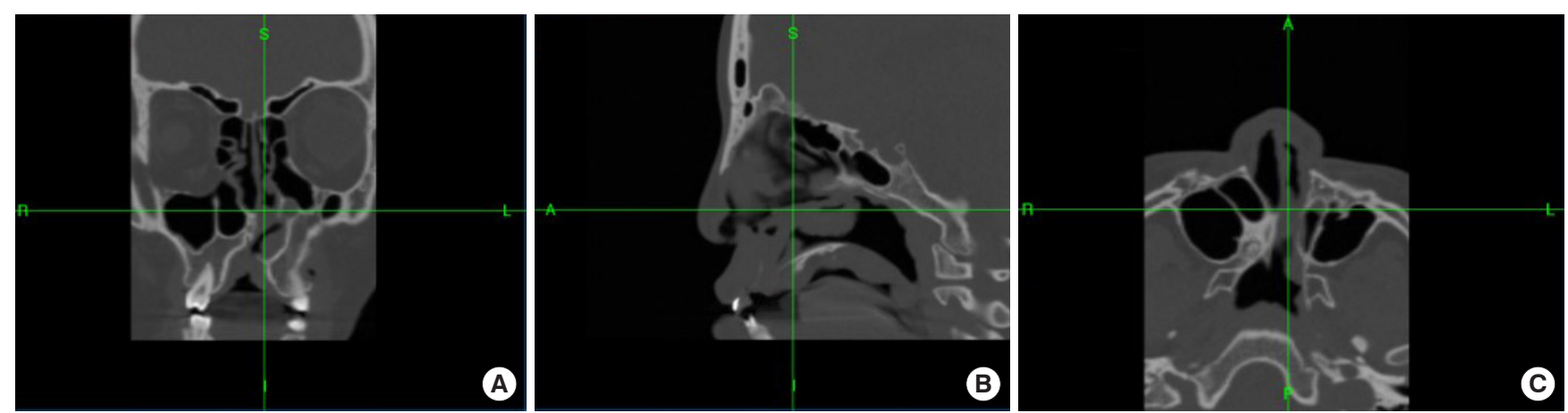

Fig. 3. View on the image-guided navigation system during surgery. (A) Coronal view. (B) Sagittal view. (C) Axial view. A, anterior; P, posterior; S, superior; I, inferior; R, right; L, left.

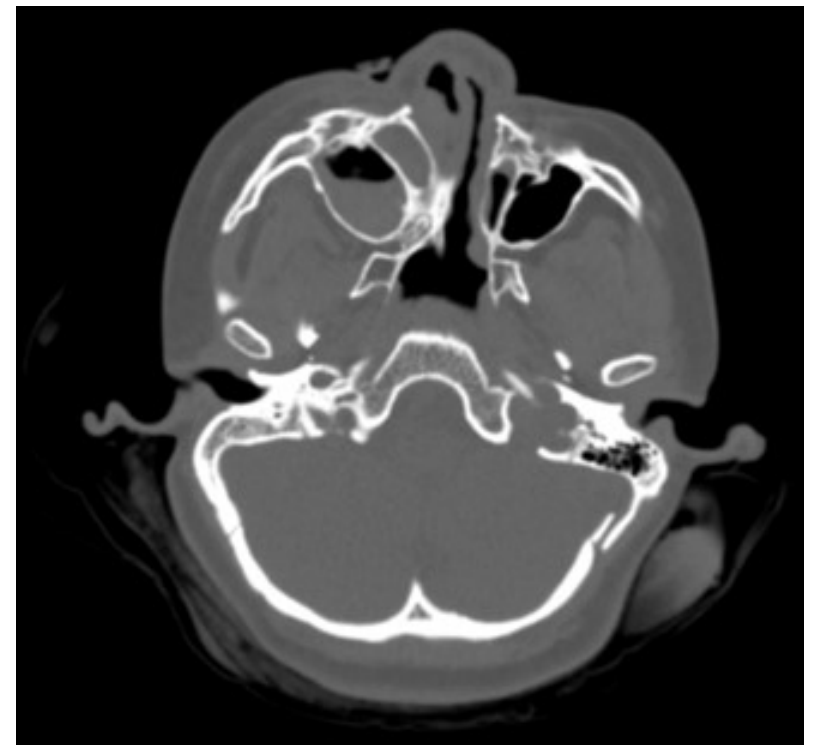

Fig. 4. Postoperative computed tomographic scan 1 year after surgery showing no evidence of restenosis in the left nasal airway.

idence of restenosis was found over the course of 26 months of follow-up with serial intranasal endoscopy and CT scans (Fig. 4). Her pronunciation was not clear due to rhinolalia clausa, which remained present after surgery, but improved after sustained speech practice. The moderate hyposmia that was present before surgery slightly improved postoperatively. The patient can now breathe nasally and reports that it is easier for her to breathe.

\section{DISCUSSION}

The present case is the first report of bilateral choanal atresia found in adulthood in a patient with a Tessier number 3 facial cleft. The coexistence of craniofacial cleft and choanal atresia is rare, and has only been reported in a few cases, especially in patients with bilateral choanal atresia. Bonafos et al. [11] reported three cases of unilateral choanal atresia and Tessier number $1 / 13$ and $2 / 12$ facial cleft in newborns. Three cases involving bilateral choanal atresia have been reported in the literature. Dey [2] reported a case of bilateral choanal atresia associated with a right number 4 and left number 3 facial cleft. Altuntas et al. [3] reported a case of bilateral choanal atresia with a Tessier number 2 facial cleft in a 6-year-old girl. Ragavan et al. [4] reported a case of bilateral choanal atresia with a Tessier number 3 facial cleft in a 5-day-old neonate.

Bilateral choanal atresia is rarely diagnosed in adults, as only 11 cases have been reported [6-9]. Newborns are obligate nasal breathers until mouth breathing is established with the descent of the larynx at approximately 4 to 6 weeks of life. In cases of bilateral choanal atresia, infants may present with acute respiratory distress and intermittent cyanosis that improves with crying [12]. The symptoms of bilateral choanal atresia can vary from mild difficulty in breathing during breastfeeding to severe upper airway obstruction [13]. The diagnosis of congenital bilateral atresia in adulthood remains exceptionally unusual, but it may occur in patients who are oligosymptomatic or have partial choanal atresia, causing their condition to be overlooked [8]. It is assumed that the case presented herein was neglected because the patient had mild symptoms, as she did not show any significant respiratory distress as a newborn and did not require treatment in the intensive care unit.

Craniofacial cleft and bilateral choanal atresia may share commonalities in terms of their embryological pathogenesis. Although several theories have been proposed, the etiology of choanal atresia remains controversial [5]. It is generally believed to be caused by persistence of the buccopharyngeal membranes or by failure of the oronasal membrane to rupture. Another theory is that malrotation of the burrowing nasal pits is caused by a misdirection of mesodermal flow resulting from local factors $[14,15]$.

The embryological pathogenesis of craniofacial cleft also has 
yet to be fully elucidated. Anomalies in the migration of neural crest cells were thought to be a common explanation for the pathogenesis of both craniofacial cleft and choanal atresia. In the 4th week of embryological development, the neural cells begin to migrate from the neural crests to the frontonasal process, where they form a mesenchymatous matrix that secondarily comprises the cartilaginous structures of the nasal capsule. An anomaly in the migration of those cells may occur at the origin of a rotation defect of the nasal cavity, with failure of the bucconasal membrane to rupture [11].

In present case, the diagnosis of bilateral choanal atresia was missed when the patient was born and in the first evaluation before septoplasty. Bonafos et al. [11] insisted on the importance of evaluating any patient presenting with a Tessier number $1 / 13$ or $2 / 12$ facial cleft for evidence of choanal atresia. Therefore, the possible presence of choanal atresia must be systematically investigated in craniofacial cleft patients.

For the surgical treatment of choanal atresia in cases of craniofacial cleft, the surgical procedure should be localized precisely to avoid damaging other tissues. Craniofacial cleft is associated with a risk of a cranial base anomaly with a possibility of meningoencephalocele [11]. In the present case, we localized the exact site before surgery using a lumbar puncture needle that extended from the nasal cavity under endoscopic guidance. Furthermore, we utilized an IGNS to ensure that we performed surgery at precisely the exact site.

In summary, this report describes the first case of bilateral choanal atresia found in an adult patient with a Tessier number 3 facial cleft. The patient underwent successful surgery using an IGNS. This report may provide further evidence for an association between choanal atresia and craniofacial cleft. It is important to systematically investigate the possibility of choanal atresia in craniofacial cleft patients. For craniofacial cleft patients affected by choanal atresia, an IGNS can be a surgical option for finding the exact anatomical site for surgery and ensuring patient safety.

\section{NOTES}

\section{Conflict of interest}

No potential conflict of interest relevant to this article was reported.

\section{Ethical approval}

The study was approved by the Institutional Review Board of Pusan National University Medical Center (IRB No. H-1905-041079) and performed in accordance with the principles of the Declaration of Helsinki. Written informed consent was obtained.

\section{Patient consent}

The patient provided written informed consent for the publication and the use of her images.

\section{ORCID}

Ji Yoon Sung https://orcid.org/0000-0001-6925-8120

Kyu-Sup Cho https://orcid.org/0000-0002-4381-6996

Yong Chan Bae https://orcid.org/0000-0002-0268-4667

Seong Hwan Bae https://orcid.org/0000-0002-7203-8978

\section{REFERENCES}

1. Tessier P. Anatomical classification facial, cranio-facial and latero-facial clefts. J Maxillofac Surg 1976;4:69-92.

2. Dey DL. Oblique facial clefts. Plast Reconstr Surg 1973;52:25863.

3. Altuntas A, Yilmaz MD, Kahveci OK, Derekoy S, Yucel A. Coexistence of choanal atresia and Tessier's facial cleft number 2. Int J Pediatr Otorhinolaryngol 2004;68:1081-5.

4. Ragavan M, ArunKumar S, Balaji NS. Bilateral choanal atresia with Tessier type 3 facial cleft: a rare association. J Neonatal Surg 2012;1:48

5. Cinnamond MJ. Congenital anomalies of the nose. In: ScottBrown WG, Kerr AG, Gleeson M, Stephens D, Booth JB, Mackay IS, et al., editors. Scott-Brown's otolaryngology. 6th ed. Oxford: Butterworth-Heinemann; 1997. p. 1-10.

6. Tinoco P, Pereira JCO, Filho RCL, Brito TSC, Pereira BM, Carrara VL, et al. Bilateral choanal atresia in 34 year-old patients. Int Arch Otorhinolaryngol 2010;14:481-4.

7. Chaudhary N, Jain A, Kapoor R, Motwani G. Bilateral complete choanal atresia in an adult woman: managed with nasal endoscopes. J Indian Med Assoc 2010;108:109-10.

8. Anajar S, Hassnaoui J, Rouadi S, Abada R, Roubal M, Mahtar M. A rare case report of bilateral choanal atresia in an adult. Int J Surg Case Rep 2017;37:127-9.

9. Carpenter RJ, Neel HB 3rd. Correction of congenital choanal atresia in children and adults. Laryngoscope 1977;87:1304-11.

10. Eliashar R, Sichel JY, Gross M, Hocwald E, Dano I, Biron A, et al. Image guided navigation system: a new technology for complex endoscopic endonasal surgery. Postgrad Med J 2003;79: 686-90.

11. Bonafos G, Capon-Degardin N, Fayoux P, Pellerin P. Choanal atresia and rare craniofacial clefts: report of three cases with a review of the literature. Cleft Palate Craniofac J 2004;41:78-83.

12. Kwong KM. Current updates on choanal atresia. Front Pediatr 2015;3:52.

13. Voegels RL, Chung D, Lessa MM, Lorenzetti FT, Goto EY, Butugan $\mathrm{O}$. Bilateral congenital choanal atresia in a 13-year-old 
patient. Int J Pediatr Otorhinolaryngol 2002;65:53-7.

14. Admiraal RJ, Joosten FB, Huygen PL. Temporal bone CT findings in the CHARGE association. Int J Pediatr Otorhinolaryngol 1998;45:151-62.
15. Hengerer AS, Strome M. Choanal atresia: a new embryologic theory and its influence on surgical management. Laryngoscope 1982;92(8 Pt 1):913-21. 